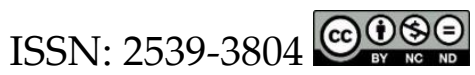

\title{
Lectura académica en la formación universitaria: tendencias en investigación ${ }^{1}$
}

Emilce Moreno Mosquera

Pontificia Universidad Javeriana

Bogotá, Colombia

\section{Resumen}

En este artículo se analiza literatura sobre lectura académica, específicamente libros y artículos de investigación publicados entre 2004 y 2017 a nivel nacional e internacional. Se trata de identificar qué tendencias investigativas hay en estos trabajos, así como reconocer cuáles son los aportes teóricos de las tradiciones discursivas que abordan el tema. Inicialmente, se contextualiza el marco de estudio retomando la alfabetización académica, la literacidad académica y la literacidad disciplinaria. Segundo, se explica la ruta metodológica que orientó la revisión bibliográfica. Tercero, se argumenta en torno a los hallazgos y se plantean algunas líneas de investigación que quedan abiertas.

Palabras clave: lectura académica; alfabetización; literacidad académica; literacidad disciplinaria; revisión de literatura.

\begin{abstract}
Academic Reading in University Education: Tendencies in Research

In this article, we analyze literature on academic reading, specifically, books and articles published between 2004 and 2017 at national and international level. The aim is to identify the research trends underlying those academic works. It also aims at recognizing what are the theoretical contributions of the discursive traditions that address the issue. Initially, the study framework is contextualized around literacy, academic literacy and disciplinary literacy. Secondly, the methodological route that guided

\footnotetext{
${ }^{1}$ Este artículo hace parte del proyecto de investigación doctoral titulado "La lectura en la formación universitaria. Análisis de prácticas de enseñanza y aprendizaje de lectores de campos disciplinares específicos", llevado a cabo por la autora en el marco del Doctorado Interinstitucional en Educación de la Universidad Pedagógica Nacional. El proyecto es financiado por la Pontificia Universidad Javeriana.
} 
the bibliographic review is explained. Finally, the findings are discussed and some research lines are considered.

Key words: academic reading; literacy; academic literacy; disciplinary literacy; literature review.

\section{Résumé}

\section{La lecture académique dans les études universitaires : tendances en recherche}

Dans cet article on analyse la littérature sur la lecture académique, plus précisément des ouvrages et des articles publiés entre 2004 et 2017 aux niveaux national et international. On analyse les tendances de la recherche dans ces publications et les apports théoriques des traditions discursives dans lequelles ce sujet est traité. Premièrement, on contextualise cette étude dans l'alphabétisation académique, la littératie académique et la littératie disciplinaire. Deuxièmement, on explique le parcours méthodologique qui a guidé la revue de la littérature. Enfin, on présente des arguments sur les résultats obtenus et on suggère quelques pistes de recherches future.

Mots-clés : lecture ; alphabétisation académique ; littératie ; littératie disciplinaire ; revue de littérature. 


\section{Cómo CITAR ESTE ARTÍcUlO}

Moreno Mosquera, E. (2019). Lectura académica en la formación universitaria: tendencias en investigación. Lenguaje, 47(1), 91-119. doi: 10.25100/lenguaje.v47i1.7180 


\section{CONSIDERACIONES INICIALES A NIVEL TEÓRICO Y METODOLÓGICO}

La lectura en la universidad viene siendo objeto de estudio de numerosas investigaciones desde hace más de tres décadas en el contexto internacional. En Latinoamérica ha cobrado en la última década una gran visibilidad, lo cual ha permitido que se puedan identificar algunas tendencias y modelos. La lectura junto con la escritura "se aprenden en ocasión de enfrentar las prácticas de producción discursiva y consulta de textos propias de cada materia, y según la posibilidad de recibir orientación y apoyo por parte de quien domina y participa de estas prácticas" (Carlino, 2002, p. 7). En ese sentido, esto implica el dominio de modos de leer y escribir particulares de cada cultura disciplinaria. De ahí la necesidad de enmarcar este trabajo en las tradiciones discursivas que han cobrado importancia en el estudio de las prácticas de lectura y escritura académicas, en las que lo disciplinario se ha ido constituyendo en una variable fundamental. Específicamente en este artículo se propone caracterizar y analizar el aporte de artículos de investigación y libros de investigación publicados entre 2004 y 2017, que aporten a la conceptualización sobre la lectura en la universidad. En concordancia con ello, las preguntas que orientan esta indagación son: ¿Cuál es la naturaleza de la investigación sobre lectura académica desde 2004 hasta 2017 publicada en libros y artículos en revistas especializadas?, ¿Cuáles son las tendencias de investigación en torno a la lectura en el ámbito universitario en los últimos diez años?, ¿Qué perspectivas de investigación se abren en este campo?

\section{Tradiciones de estudio: literacidad académica, alfabetización académica y literacidad disciplinaria}

Esta investigación se encuentra inserta en una discusión actual en torno a dos tradiciones discursivas principalmente: literacidad académica y alfabetización académica. Habría una tercera denominada literacidad disciplinaria. Según Barton y Hamilton (1990), el término literacidad tomó fuerza en el ámbito anglosajón desplazando a los de lectura y escritura en las décadas de los setenta y ochenta. La concepción en torno a literacidad o literacidades surgió en el Reino Unido, como una propuesta que incluye eventos mediados por textos escritos implicados en diferentes contextos sociales, no únicamente el académico. De acuerdo con Lea y Street $(1998,2006)$ la escritura y la literacidad en contextos académicos podrían conceptualizarse mediante tres perspectivas interrelacionadas: I. Modelo de habilidades de estudio; II. Modelo de socialización académica, y III. Modelo de literacidades académicas. Sobre estos modelos Lea y Street (2006) explican lo siguiente: 
Los tres modelos no son mutuamente excluyentes; más bien, coexisten. Los tres modelos se podrían aplicar a cualquier contexto académico, tales como examinar la escritura y las prácticas de literacidad en biología, antropología o en la formación del profesorado y de cómo los estudiantes llegan a entender y usar esas prácticas de literacidad en cada contexto académico. También hay coexistencia a nivel teórico. Por ejemplo, tanto el modelo de socialización académica, como el de literacidades académicas se centran en la relación entre epistemología y actos de escritura y literacidad en áreas temáticas y disciplinas (Bazerman, 1988; Berkenkotter y Huckin, 1995). Sin embargo, el modelo de literacidad académica va más lejos al centrarse en la relación de la epistemología y la escritura, no sólo en el área temática en general, sino también en los requisitos institucionales (p.e. en relación con el plagio, la retroalimentación); así como, en contextos más específicos como la variación entre las necesidades de los miembros de la facultad a nivel individual e incluso las tareas individuales de los estudiantes. (p. 369).

El primer modelo estaría más centrado en la parte gramatical sin tener en cuenta el contexto, mientras en los otros dos modelos sería importante la relación entre epistemología y escritura, pero, como se señala, con una orientación diferente. De hecho, el tercer modelo se relaciona con los Nuevos Estudios de Literacidad (NEL), los cuales emplean métodos etnográficos para describir y analizar los usos de los textos escritos. Desde este modelo, las prácticas de literacidad son históricas, organizadas por instituciones sociales y por relaciones de poder (Barton y Hamilton, 2000). Además, "la literacidad siempre implica una manera de usar la lectura y la escritura en el marco de un propósito social específico" (Zavala, 2009a, p. 23). Algunos de los trabajos que se enmarcan desde este modelo son los de Barton y Hamilton $(1998,2000)$, Street (1984, 1993) en Inglaterra, y Gee (1990) en Estados Unidos. Así mismo, están los de Cassany (2006, 2009); Aliagas, Castellà y Cassany (2009); Kalman (2004); Montes y López-Bonilla (2017); Peredo Merlo (2002); Zavala (2002); Zavala, Niño-Murcia y Ames (2004); en el ámbito hispanoamericano, entre otros.

Desde este modelo, existen trabajos que reconocen la importancia del contexto en el que se dan las prácticas de lectura (Barton, 1994; Cassany, 2009; Cassany y Morales, 2009; Cook-Gumperz, 1988; Gee, 2001; González de la Torre, 2013; Lea y Street, 2006; Peredo Merlo, 2002; Rockwell y Mercado, 1988; Street, 2004; Verhoeven, 1994; Zavala, 2009). Trabajos que argumentan a favor de la lectura ubicada en un contexto histórico y cultural, lo cual supone comprender que la literacidad es social y no se ubica sólo en la mente de quienes leen o en los textos, sino en la interacción de los sujetos implicados y en lo que ellos hacen con los textos.

De otra parte, en cuanto a la alfabetización académica, Carlino (2013a) explica que es "un proceso de enseñanza que puede (o no) ponerse en marcha para favorecer el 
acceso de los estudiantes a las diferentes culturas escritas de las disciplinas" (p. 370). Se relaciona, entonces, con las acciones didácticas de los docentes por enseñar a exponer, argumentar, resumir, etc. a sus estudiantes bajo el auspicio de la institución en la cual se encuentran ubicados y de acuerdo con los marcos que impone cada asignatura. Según esta autora, esto implica dos objetivos relacionados con enseñar a participar en los géneros propios de un campo del saber y enseñar las prácticas de estudio adecuadas para aprender en él. Estos objetivos se relacionan: "en el primer caso, se trata de formar para escribir y leer como lo hacen los especialistas; en el segundo, de enseñar a leer y a escribir para apropiarse del conocimiento producido por ellos" (p. 370). De acuerdo con lo anterior, fomentar la participación de los estudiantes en prácticas discursivas contextualizadas conlleva a que los docentes de las diferentes asignaturas estén implicados, indiferentemente de si son o no expertos en lengua materna; además, desde la alfabetización académica no se busca remediar la formación de aquellos que ingresan a la universidad.

De acuerdo con lo anterior, la alfabetización pone el acento en el quehacer educativo, en la caracterización de las prácticas docentes, en las interacciones en el aula en torno al trabajo de comprensión y producción textual. En este modelo se inscriben, entre otros, trabajos como Carlino (2001, 2002, 2003a, 2003b, 2004, 2005, 2007, 2009, 2012, 2013a); Di Benedetto y Carlino (2007); Fernández y Carlino (2010), así como los trabajos de los diferentes miembros del Grupo para la Inclusión y Calidad Educativas a través de Ocuparnos de la Lectura y la Escritura en todas las Materias (GICEOLEM); Castelló (2007, 2009); Cisneros, Olave y Rojas (2013); Narváez (2012); Narvaja de Arnoux, Di Stefano y Pereira (2002); Parodi (2010); Pérez y Rincón (2013); Quintana, García-Arroyo, Arribas y Hernández (2010); Mateos y Solé (2009); entre otros.

Sobre estas dos líneas de investigación, Carlino (2013a) explica lo siguiente:

Si bien mantienen relaciones, ninguno de estos campos de investigación ni los conocimientos emergentes de ellos, pueden reducirse a o derivarse del otro. En este sentido, distinguir alfabetización de literacidad permite diferenciar no sólo dos conceptos, sino dos categorías de problemas teóricos y dos clases de investigación: la didáctica-educativa y la lingüística-etnográfica. (p. 373).

También existe otro campo muy pertinente para el presente estudio, al cual se denomina Literacidad disciplinaria, cuya premisa consiste en que el conocimiento de las disciplinas es siempre discursivo y mediado por textos; en esa medida, conviene examinar las formas de producir ese conocimiento por vía oral y escrita en cada disciplina (Moje, 2007). Algunos aspectos que caracterizan esta literacidad son: I. enfatiza en las actividades que permiten que los textos sean relevantes y adquieran sentido; II. Intenta que a través de la participación en actividades similares a las que realizan los especialistas de cada disciplina, los estudiantes logren aprender de manera 
profunda los conocimientos disciplinarios y las epistemologías para que puedan transformar y producir nuevo conocimiento; III. Comprende dos términos claves: participación y práctica (Moje, 2007). Lo anterior implica identificar las prácticas de la disciplina presentes en el aula de clase; igualmente, saber cómo instrumentarlas como objetivos de aprendizaje en el currículo y como actividades en el aula (Ford y Forman, 2006). Especialmente los trabajos que se han desarrollado desde esta perspectiva corresponden a la educación secundaria y abordan las prácticas de literacidad en el marco de un dominio determinado (Driver, Asoko, Leach, Mortimer y Scott, 1994; Fang, 2012; Ford y Forman, 2006; Moje, 2007, 2008; Stockdill, Learned y Moje, 2011; Wineburg, 1991, 1998, 2001).

\section{MÉTODO: RECOLECCIÓN DE DATOS Y ASPECTOS PROCEDIMENTALES}

En este apartado se explica el proceso de revisión y análisis del corpus seleccionado, el cual se dio en tres etapas: 1) Revisión de las bases de datos para seleccionar artículos de investigación. 2) Desarrollo de una plantilla de revisión analítica, que sirva para documentar y describir los temas y las cualidades de la investigación en torno al objeto de estudio. 3) Construcción de una ruta argumental que permita analizar la naturaleza y las tendencias en investigación identificadas.

\section{Revisión de las bases de datos}

Se hizo una búsqueda bibliográfica de cinco bases de datos electrónicas de los años 2004-2017: ERIC (EBSCO), ProQuest, Redalyc, Scielo y Wiley Online Library. Los términos de la búsqueda fueron "lectura académica", "lectura y alfabetización", "lectura y literacidad", lectura en las disciplinas" y su equivalente en inglés. Se realizaron búsquedas sólo de artículos que reportaran investigaciones realizadas en el contexto universitario, revisados por pares; se excluyeron: disertaciones, documentos no publicados, informes y reseñas de libros. Se ubicaron 33 artículos de investigación, 2 libros de investigación y un capítulo, los cuales fueron objeto de análisis.

\section{Plantilla de revisión analítica}

A partir de la elaboración de las fichas de lectura de los artículos, en la plantilla se identificaron los siguientes aspectos: año de publicación/número de artículos, país, tipo de publicación y, teniendo como base lo identificado en la naturaleza de los artículos de investigación revisados, se encontraron seis tendencias frecuentes: 1) Revisión de la literatura existente. Estados del arte precedentes; 2) Investigación interinstitucional. El caso de la investigación ¿Para qué se lee y se escribe en la universidad colombiana? Un 
aporte a la consolidación de la cultura académica del país y los productos derivados; 3) Investigaciones asociadas a la alfabetización académica; 4) Investigaciones asociadas a la literacidad académica; 5) Investigaciones sobre función epistémica de la lectura; 6) Investigaciones sobre hábitos y estrategias de lectura. A continuación, se presenta la Tabla 1, que resume los artículos encontrados:

Tabla 1. Resumen de los artículos encontrados en las bases de datos

\begin{tabular}{|c|c|c|c|c|}
\hline $\begin{array}{l}\text { No. de } \\
\text { artículos }\end{array}$ & $\begin{array}{c}\text { Año de } \\
\text { publicación }\end{array}$ & País & $\begin{array}{c}\text { Tipo de } \\
\text { publicación }\end{array}$ & Tendencia \\
\hline 1 & 2017 & México & $\begin{array}{l}\text { Artículo de } \\
\text { investigación }\end{array}$ & $\begin{array}{l}\text { Investigaciones asociadas a la } \\
\text { literacidad académica }\end{array}$ \\
\hline 1 & 2016 & Colombia & $\begin{array}{l}\text { Artículo de } \\
\text { investigación }\end{array}$ & $\begin{array}{l}\text { Investigaciones asociadas a la } \\
\text { literacidad académica }\end{array}$ \\
\hline 1 & 2016 & México & $\begin{array}{l}\text { Artículo de } \\
\text { investigación }\end{array}$ & $\begin{array}{l}\text { Investigaciones sobre función } \\
\text { epistémica de la lectura }\end{array}$ \\
\hline 1 & 2016 & Colombia & $\begin{array}{l}\text { Artículo de } \\
\text { investigación }\end{array}$ & $\begin{array}{l}\text { Investigaciones sobre función } \\
\text { epistémica de la lectura }\end{array}$ \\
\hline 1 & 2016 & $\begin{array}{l}\text { Chile y } \\
\text { Estados } \\
\text { Unidos }\end{array}$ & $\begin{array}{l}\text { Artículo de } \\
\text { investigación }\end{array}$ & $\begin{array}{l}\text { Investigaciones sobre función } \\
\text { epistémica de la lectura }\end{array}$ \\
\hline 1 & 2015 & Colombia & $\begin{array}{l}\text { Artículo de } \\
\text { investigación }\end{array}$ & $\begin{array}{l}\text { Investigaciones asociadas a la } \\
\text { alfabetización académica }\end{array}$ \\
\hline 1 & 2014 & México & $\begin{array}{l}\text { Artículo de } \\
\text { investigación }\end{array}$ & $\begin{array}{l}\text { Investigaciones sobre hábitos } \\
\text { de y estrategias de lectura }\end{array}$ \\
\hline 1 & 2013 & Argentina & Estado del arte & $\begin{array}{l}\text { Revisión de literatura } \\
\text { existente }\end{array}$ \\
\hline 1 & 2013 & Colombia & $\begin{array}{l}\text { Artículo de } \\
\text { investigación }\end{array}$ & $\begin{array}{l}\text { Investigación } \\
\text { interinstitucional }\end{array}$ \\
\hline 1 & 2012 & Colombia & $\begin{array}{l}\text { Artículo de } \\
\text { investigación }\end{array}$ & $\begin{array}{l}\text { Investigación } \\
\text { interinstitucional }\end{array}$ \\
\hline 1 & 2012 & Holanda & $\begin{array}{l}\text { Artículo de } \\
\text { investigación }\end{array}$ & $\begin{array}{l}\text { Investigaciones sobre función } \\
\text { epistémica de la lectura }\end{array}$ \\
\hline 1 & 2011 & Colombia & Estado del arte & $\begin{array}{l}\text { Revisión de literatura } \\
\text { existente }\end{array}$ \\
\hline 1 & 2009-2011 & Colombia & $\begin{array}{l}\text { Libro de } \\
\text { investigación }\end{array}$ & $\begin{array}{l}\text { Investigación } \\
\text { interinstitucional }\end{array}$ \\
\hline 1 & 2011 & Colombia & $\begin{array}{l}\text { Artículo de } \\
\text { investigación }\end{array}$ & $\begin{array}{l}\text { Investigación } \\
\text { interinstitucional }\end{array}$ \\
\hline
\end{tabular}




\begin{tabular}{|c|c|c|c|c|}
\hline 1 & 2011 & Argentina & $\begin{array}{l}\text { Artículo de } \\
\text { investigación }\end{array}$ & $\begin{array}{l}\text { Investigaciones asociadas a la } \\
\text { alfabetización académica }\end{array}$ \\
\hline 1 & 2011 & México & $\begin{array}{l}\text { Artículo de } \\
\text { investigación }\end{array}$ & $\begin{array}{l}\text { Investigaciones asociadas a la } \\
\text { literacidad académica }\end{array}$ \\
\hline 1 & 2011 & Argentina & $\begin{array}{l}\text { Artículo de } \\
\text { investigación }\end{array}$ & $\begin{array}{l}\text { Investigaciones sobre hábitos } \\
\text { de y estrategias de lectura }\end{array}$ \\
\hline 3 & 2010 & Colombia & $\begin{array}{l}\text { Artículo de } \\
\text { investigación }\end{array}$ & $\begin{array}{l}\text { Investigación } \\
\text { interinstitucional }\end{array}$ \\
\hline 1 & 2010 & Colombia & $\begin{array}{l}\text { Artículo de } \\
\text { investigación }\end{array}$ & $\begin{array}{l}\text { Investigación } \\
\text { interinstitucional }\end{array}$ \\
\hline 1 & 2010 & Colombia & $\begin{array}{l}\text { Artículo de } \\
\text { investigación }\end{array}$ & $\begin{array}{l}\text { Investigación } \\
\text { interinstitucional }\end{array}$ \\
\hline 1 & 2010 & Argentina & $\begin{array}{l}\text { Artículo de } \\
\text { investigación }\end{array}$ & $\begin{array}{l}\text { Investigaciones asociadas a la } \\
\text { alfabetización académica }\end{array}$ \\
\hline 1 & 2009 & Argentina & $\begin{array}{l}\text { Artículo de } \\
\text { investigación }\end{array}$ & $\begin{array}{l}\text { Investigaciones asociadas a la } \\
\text { alfabetización académica }\end{array}$ \\
\hline 1 & 2009 & Colombia & $\begin{array}{l}\text { Artículo de } \\
\text { investigación }\end{array}$ & $\begin{array}{l}\text { Investigaciones asociadas a la } \\
\text { alfabetización académica }\end{array}$ \\
\hline 1 & 2009 & España & $\begin{array}{l}\text { Capítulo de libro } \\
\text { de investigación }\end{array}$ & $\begin{array}{l}\text { Investigaciones asociadas a la } \\
\text { literacidad académica }\end{array}$ \\
\hline 1 & 2009 & España & $\begin{array}{l}\text { Libro de } \\
\text { investigación }\end{array}$ & $\begin{array}{l}\text { Investigaciones asociadas a la } \\
\text { literacidad académica }\end{array}$ \\
\hline 1 & 2009 & $\begin{array}{l}\text { Estados } \\
\text { Unidos }\end{array}$ & $\begin{array}{l}\text { Artículo de } \\
\text { investigación }\end{array}$ & $\begin{array}{l}\text { Investigaciones sobre función } \\
\text { epistémica de la lectura }\end{array}$ \\
\hline 1 & 2008 & España & $\begin{array}{l}\text { Artículo de } \\
\text { investigación }\end{array}$ & $\begin{array}{l}\text { Investigaciones asociadas a la } \\
\text { alfabetización académica }\end{array}$ \\
\hline 1 & 2008 & India & $\begin{array}{l}\text { Artículo de } \\
\text { investigación }\end{array}$ & $\begin{array}{l}\text { Investigaciones sobre hábitos } \\
\text { de y estrategias de lectura }\end{array}$ \\
\hline 1 & 2007 & España & $\begin{array}{l}\text { Artículo de } \\
\text { investigación }\end{array}$ & $\begin{array}{l}\text { Investigaciones asociadas a la } \\
\text { alfabetización académica }\end{array}$ \\
\hline 1 & 2007 & México & $\begin{array}{l}\text { Artículo de } \\
\text { investigación }\end{array}$ & $\begin{array}{l}\text { Investigaciones sobre función } \\
\text { epistémica de la lectura }\end{array}$ \\
\hline 1 & 2006 & Colombia & $\begin{array}{l}\text { Artículo de } \\
\text { investigación }\end{array}$ & $\begin{array}{l}\text { Investigaciones asociadas a la } \\
\text { literacidad académica }\end{array}$ \\
\hline 1 & 2005 & España & $\begin{array}{l}\text { Artículo de } \\
\text { investigación }\end{array}$ & $\begin{array}{l}\text { Investigaciones asociadas a la } \\
\text { alfabetización académica }\end{array}$ \\
\hline 1 & 2005 & Argentina & $\begin{array}{l}\text { Artículo de } \\
\text { investigación }\end{array}$ & $\begin{array}{l}\text { Investigaciones asociadas a la } \\
\text { alfabetización académica }\end{array}$ \\
\hline 1 & 2004 & Argentina & $\begin{array}{l}\text { Artículo de } \\
\text { investigación }\end{array}$ & $\begin{array}{l}\text { Investigaciones asociadas a la } \\
\text { alfabetización académica }\end{array}$ \\
\hline
\end{tabular}

Fuente: Elaboración propia 
Revisión de la literatura existente. Estados del arte precedentes

Se encontraron artículos de revisión que daban cuenta de experiencias de investigación y publicaciones sobre lectura (y escritura) académica (Carlino, 2013a; Uribe y Camargo, 2012). El trabajo de Carlino (2013a) se constituye en un antecedente relevante para la presente investigación; específicamente se da cuenta de experiencias, iniciativas pedagógicas y publicaciones sobre la enseñanza de la lectura y la escritura en la universidad (en el contexto argentino especialmente), pero mostrando la influencia de diferentes tradiciones investigativas, tales como "escribir a través del currículum" (Bazerman et al. 2005; Russell, 2002), "enseñar escritura en contexto" (Chalmers y Fuller, 1996). Igualmente, se reflexiona y se actualiza la conceptualización en torno a la alfabetización académica, que es "un proceso de enseñanza que puede (o no) ponerse en marcha para favorecer el acceso de los estudiantes a las diferentes culturas escritas de las disciplinas" (Carlino, 2013a, p. 370). El acento no sólo se pone en los estudiantes, sino también en los docentes, las instituciones y la didáctica-educativa, que articulados podrían ir más allá de la fragmentación de las prácticas de lectura y escritura y la inscripción a cursos remediales, para constituirse en espacios que posibiliten el acceso de los estudiantes a culturas letradas de diferentes disciplinas.

100 También, se encuentra el trabajo de Uribe y Camargo (2012), en el cual se presenta el análisis de algunas prácticas de lectura y escritura académicas en la universidad colombiana, divulgadas por diversos medios (bases de datos, memorias en páginas web y en CD-ROM de congresos, coloquios, encuentros y seminarios), ${ }^{2}$ un total de 40 trabajos. En el análisis se presentan las tendencias en relación al predominio de tener como objeto de estudio a los estudiantes, los tópicos en los que se centran las investigaciones y los enfoques asociados a la lectura y la escritura más predominantes. En esta investigación se encontró que el 77 \% de los trabajos revisados enfatizó en el estudiante, lo que muestra que las investigaciones se centran en el déficit. El resto se centra en el profesor como responsable del aprendizaje. Entre las problemáticas identificadas están: el diagnóstico sobre el estado de los procesos de lectura y escritura (32\%); la preocupación por el uso de estrategias cognitivas y metacognitivas (15\%); el desarrollo de competencias (10\%), los aspectos actitudinales (8\%) y la motivación (3\%). En relación con las perspectivas teóricas que predominan en los informes de investigación están: el enfoque lingüístico textual y el análisis del discurso (60\%), el enfoque sociocultural (10\%), el cognitivo (8\%), el psicolingüístico (7\%), el constructivista $(5 \%)$, el semántico-comunicativo $(3 \%)$ y otros enfoques (7\%). Al finalizar, las autoras explican que el análisis les permitió: “objetivar algunas

\footnotetext{
${ }^{2}$ Esta revisión se enmarca en la investigación interinstitucional financiada por Colciencias ¿Para qué se lee y escribe en la universidad colombiana? Un aporte a la consolidación de la cultura académica.
} 
percepciones subjetivas, definir carencias, posibilidades y desafíos, mediante caminos propuestos por los docentes investigadores que, a su vez, provocan cuestionamientos en relación con el deber ser de las prácticas académicas" (Uribe y Camargo, 2012, p. 338).

Investigación interinstitucional. El caso de la investigación ¿Para qué se lee y se escribe en la universidad colombiana? Un aporte a la consolidación de la cultura académica del país y los productos derivados

Pérez y Rincón (2013) entre 2009 y 2011 reunieron 17 equipos de investigación de diferentes universidades para estudiar las prácticas de lectura y escritura en la universidad y su relación con cierto tipo de cultura académica. La hipótesis que orientaba este estudio era:

El tipo de prácticas académicas que promueve la universidad y que se concreta en las prácticas pedagógicas y didácticas de sus docentes, produce ciertos modos de leer y escribir en los estudiantes, y esto se relaciona de modo directo con cierto tipo de cultura académica. (pp. 39-40).

A través del análisis de diferentes fuentes -syllabus, encuestas, documentos de política institucional, grupos de discusión, etc.- hicieron una descripción interpretativa de las prácticas de lectura y escritura de los estudiantes universitarios de las universidades involucradas, desde una perspectiva pedagógica y didáctica, que reconoce además que la comprensión de dichas prácticas se relaciona de modo directo con el tipo de cultura académica en la cual se inscribe. Específicamente, se analizaron 20 casos de prácticas destacadas de enseñanza de las universidades involucradas. Entre las conclusiones más importantes de este estudio se encuentran:

- Si bien no se identifican unas directrices explicitas de políticas institucionales, sí hay preocupación por el tema de la lectura y la escritura académicas. Existe un número considerable de cursos basados en una concepción de lectura y escritura como competencias generales, que no son una condición suficiente para el ingreso a la cultura académica universitaria, en la que se reconozca la función epistémica de la lectura y la escritura, esto es, conectadas a las necesidades y a modos específicos de uso para construir y aprender contenidos en una disciplina específica.

- Se hace necesario considerar el hecho de que los apuntes de clase sean el texto más leído y escrito por los estudiantes, dado que es fundamental que cualquier 
profesional en formación aprenda a consultar fuentes primarias y se vincule a su comunidad de práctica.

- La universidad, en sus políticas, y los docentes, en sus prácticas de enseñanza, no tienen como propósitos explícitos fortalecer la lectura y la escritura académica con funciones epistémicas, es decir, "no es explícito que se planifiquen, realicen y evalúen unas determinadas configuraciones y secuencias didácticas con el fin de orientar modos específicos de leer y escribir, propios de la disciplina" (p. 280). Esto a su vez, confirma que se promueven usos de lectura y escritura, orientados principalmente a responder tareas y evaluaciones. Así mismo, la necesidad de centrar la reflexión al respecto en la didáctica, la cual permitiría comprender la lectura y la escritura como prácticas sociales (Pérez y Rincón, 2013).

De este trabajo se derivaron diferentes artículos, como: Rincón y Gil (2010); Quintero y Duque (2010); Chois y Santacruz (2010); García-Vera (2011); Uribe y Camargo (2012); Narváez y Chois (2012); Pérez y Rodríguez (2013), en los cuales se presentan resultados parciales de cada experiencia investigativa.

\section{Investigaciones asociadas a la alfabetización académica}

Como se explicó inicialmente, existen dos campos de investigación: alfabetización y literacidad académica. En el primer campo, algunas de las investigaciones que se ocupan de la lectura son: Carlino (2005, 2009); Estienne y Carlino (2004); Fernández y Carlino (2010); Cartolari y Carlino (2011); Solé et al. (2005); Mateos, Villalón, de Dios y Martin (2007); Mateos, Martin, Pecharromán, Luna y Cuevas (2008); Narváez y Cadena (2009); Quintana et al. (2010); Salazar et al. (2015).

Escribir, leer y aprender en la universidad (Carlino, 2005) se constituye en una obra fundamental para el campo de la alfabetización académica, cuyas preguntas centrales son: ¿Cómo se relaciona la escritura y la lectura con el aprendizaje? ¿De qué modo pueden los docentes sacar provecho de estas relaciones en beneficio de la formación de los estudiantes? ¿Qué es y qué no es la alfabetización académica? A lo largo de cuatro capítulos se analiza el valor epistémico de la lectura y la escritura, las cuales implican acompañamiento docente y condiciones didácticas favorables. Así mismo, se aborda el modo en que los docentes pueden promover el aprendizaje a través de situaciones de evaluación, que suelen incluir lectura y escritura. Esto en el marco de situaciones que permitan constituir dichas prácticas en objetos de enseñanza al interior de las asignaturas. En esa misma línea están los estudios de Estienne y Carlino (2004); Carlino (2011); Cartolari y Carlino (2011). El primero presenta un estudio exploratorio en un curso sobre prácticas de lectura en carreras universitarias de las ciencias sociales, para lo cual analizaron entrevistas extensas a tres estudiantes y a tres profesores, además de otros documentos. A partir de lo expresado por estos, se encuentra que a nivel 
universitario se parte del supuesto de que el estudiante es adulto y puede leer de modo autónomo, lo cual es discutido y se argumenta sobre la necesidad de plantear ayudas pedagógicas al respecto.

La investigación de Carlino (2011) se enmarca en un estudio más grande, ${ }^{3}$ que surge para ampliar los datos; se analiza cuáles son las perspectivas de estudiantes y docentes acerca de las tareas de lectura y escritura que se llevan a cabo en materias de las ciencias sociales y cómo realimentan los docentes los escritos de sus alumnos. Esto, además, como una iniciativa para promover la necesidad de que los docentes se ocupen de enseñar a leer y escribir en las disciplinas en la universidad. Cartolari y Carlino (2011) analizan dos situaciones que muestran modelos didácticos, que responden a distintos modos de enseñar (monológico y dialógico), en donde se fomenta o no la dialogicidad entre los intercambios orales en clase, la lectura y las notas que toman los estudiantes de dos asignaturas de historia y geografía. De manera que se puede analizar el lugar que le otorga el docente a leer para aprender, a través de la observación de aspectos como el tiempo dedicado al trabajo de lectura, trabajo de pares, realimentación de interpretaciones y el sentido que le conceden a los apuntes que toman los estudiantes.

En una línea cercana están los trabajos de Solé et al. (2005), Mateos et al. (2007) y Mateos et al. (2008). En el primero, los autores caracterizan las tareas de lectura y escritura que se proponen y hacen para aprender, por lo cual se utiliza un diseño "ex post facto" prospectivo, con tres variables independientes: rol del profesor y del alumno, nivel educativo (primer ciclo de ESO) y dominio de conocimiento; así como dos variables dependientes: frecuencia de las tareas y sus características. Esto a fin de identificar el nivel de profundidad de aprendizaje promovido por el docente y la percepción de la utilidad de las tareas (aprender, repasar, profundizar). En la misma perspectiva, como parte de un proyecto de investigación más amplio, Solé et al. (2005) tratan de determinar en qué medida los estudiantes universitarios en España utilizan la lectura y la escritura (de manera que fortalezca su función epistémica) y en qué medida esto depende de las disciplinas académicas en que se enmarca el estudio. Un estudio similar es el de Mateos et al. (2007), en el cual se identificaron las principales actividades que desarrollaban los estudiantes (conferencias, toma de notas, subrayado, resumen, esquemas, informes de práctica, etc.), actividades que no requieren un nivel alto de escritura y organización del conocimiento. Se hace la diferenciación entre estudiantes de biología, psicología e historia. Sin embargo, se advierte en la discusión que el estudio es

\footnotetext{
${ }^{3}$ Este estudio se constituyó por tres etapas de investigación: la primera, consistió en analizar a través de los borradores, cómo los estudiantes revisaban sus textos desde una perspectiva cognitiva; la segunda, consistió en comparar las revisiones de estos estudiantes, con estudiantes franceses y norteamericanos. Y, en la tercera, se desarrolló una investigación acción durante seis años, que puso a prueba las tareas de lectura y escritura en asignaturas de psicología y educación.
} 
sólo descriptivo y habría limitaciones en términos de la muestra y la fiabilidad de los datos obtenidos a través de cuestionarios. Igualmente, queda pendiente por investigar ¿en qué medida las diferencias en cuanto al uso de la lectura y la escritura de los campos disciplinarios estudiados se deben a la naturaleza particular de los métodos empleados en los diferentes dominios del conocimiento o a la conciencia de la función epistémica de estas herramientas?

Mateos et al. (2008) en la misma línea de los anteriores estudios, analizan tareas de lectura y escritura de 66 estudiantes de $1^{\circ}$ curso y 62 de $4^{\circ}$ curso de Psicología, partiendo de la premisa de que el dominio de una disciplina supone aprender a interpretar y producir sus textos. Del análisis resulta pertinente el hecho de que los estudiantes que están finalizando su formación hacen tareas de lectura y escritura más complejas, lo cual podría suponer que los estudiantes tienen más recursos y estrategias para aprender. No obstante, se advierte que sería pertinente investigar en qué medida ese incremento de tareas obedece a un aumento de la conciencia de la función epistémica que implican otros factores relacionados.

En el trabajo de Narváez y Cadena (2009) se analizan las tareas de lectura en cursos profesionales. Se parte de la premisa de que este tipo de cursos pueden ser espacios formativos de trabajo interdisciplinario, orientado por expertos en el campo de 104 la enseñanza de la lectura académica. Igualmente, se discuten algunos mitos relacionados con la lectura en la universidad, $y$, a partir de ello, se plantea una propuesta.

Quintana et al. (2010) analizan las prácticas de lectura y escritura en tres universidades de Puerto Rico para lo cual se aplicó un cuestionario abierto. A partir de los datos se encontró que hay un compromiso de integrar la alfabetización académica, para lo cual las instituciones implicadas tendrían que priorizar la lectura y la escritura en el marco del currículo. Además de esto, se debería articular la parte administrativa de las instituciones, la cual podría fortalecer la capacitación y el compromiso docente.

Salazar et al. (2015) analizan 34 experiencias de lectura y escritura en el marco de la Red de Lectura y Escritura en Educación Superior (REDLEES) entre 2008 y 2010, con el fin de hacer reconstruir la historia de las motivaciones que tuvieron diferentes universidades de incluir asignaturas y programas que fortalecieran los procesos de lectura y escritura de los estudiantes. Se concluye que hay una tendencia a diseñar asignaturas con "carácter remedial", además que se conciben estos procesos como habilidades y destrezas de lenguaje, no como prácticas sociales y culturales que forman parte de la cultura académica. 
Investigaciones asociadas a la literacidad académica

En el caso de la lectura también existen trabajos que reconocen la importancia del contexto en el que se dan las prácticas (Barton, 1994; Cassany, 2009; Cassany y Morales, 2009; Cook-Gumperz, 1988; Gee, 2001; Rockwell y Mercado, 1988; Street, 2004; Lea y Street, 2006; Verhoeven, 1994; Zavala, 2009). Trabajos que argumentan a favor de la lectura situada en un contexto histórico y cultural, lo cual supone comprender que la literacidad es social y no se ubica sólo en la mente de quienes leen o en los textos, sino en la interacción de los sujetos implicados y en lo que ellos hacen con los textos.

Si bien desde este enfoque prevalecen los estudios en el ámbito de la escritura (Hernández-Zamora, 2009; Ivanic, 1997, 1998; Jones, Turner y Street, 1999; Zavala, 2009a, entre otros), en lectura también se encuentran algunos estudios (Cassany, 2009; González de la Torre, 2011; Montes y López-Bonilla, 2017; Londoño y Bermúdez, 2017; Tejada y Vargas, 2011; Zavala, 2009b). Cassany (2009) en su libro "Para ser letrados. Voces y miradas sobre la lectura", desde las últimas teorías socioculturales plantea diferentes propuestas sobre la enseñanza de la lectura en el aula, lo cual supone poner el acento en que leer es una actividad vinculada a unas instituciones e ideologías. De modo que aprender a leer implica saber usar y relacionar los textos escritos con sus funciones sociales en la vida cotidiana. A través de quince capítulos, que incluyen ejemplos y demostraciones prácticas, se abordan diferentes prácticas de lectura relacionadas con la prensa, los artículos científicos, la multimodalidad, internet o imagen, la ideología, la lectura en voz alta, entre otros.

González de la Torre (2011) en su investigación da cuenta de cómo se configuran las prácticas de lectura de sujetos que leen en diferentes contextos: en el aula universitaria (Licenciatura de Derecho y Geografía de una universidad pública) y en el espacio laboral, desde el enfoque referido. Los resultados muestran que el contexto incide sobre la práctica, dado que se establecen rutinas de acción: "el lector [del contexto laboral] se acostumbra a leer materiales que hacen referencia a información circunstanciada, es decir, presentan en detalle los datos de una situación a la cual se enfoca el profesional en ejercicio" (pp. 48-49). En el caso de la universidad, los textos contienen información de mayor densidad y amplitud en relación con el nivel de conocimientos de los estudiantes, y en ese sentido las rutinas se hacen más exigentes. Otro estudio es el de Montes y López-Bonilla (2017), quienes presentan una revisión teórica de las nociones de literacidad, literacidad disciplinaria y alfabetización disciplinaria. A partir de esto se hace una revisión de literatura sobre la aplicación didáctica de la literacidad disciplinaria en relación con: 
Los estudios que se centran en lo que hacen los miembros de una comunidad disciplinar para acceder y producir conocimiento mediado por textos, y los estudios que analizan las características y dimensiones de los géneros disciplinares, esto último desde tres corrientes teórico-metodológicas. (p. 162).

Londoño y Bermúdez (2017) presentan algunos hallazgos sobre los niveles de literacidad en estudiantes universitarios de primeros semestres que se reportan como bajos. Se concluye que el contexto biográfico, la familia, la escuela inciden en la adquisición de competencias discursivas. En el estudio de Tejada y Vargas (2011) se plantea la importancia de integrar la literacidad funcional con la crítica. Se explica que si bien han prevalecido los estudios de la lectura y la escritura desde la psicología, la lingüística y la psicolingüística, además de la concepción de que leer y escribir son habilidades y destrezas, se hace necesario entenderlas como prácticas que se instalan en la vida social y cultural de las personas, y que pueden ser determinantes en la configuración de sociedades más justas y menos discriminatorias. De ahí que se discuta y se proponga la necesidad de "formar lectores con literacidad crítica que les ayude a configurar el ideario político y cultural" (p. 212).

Zavala (2009b) analiza el caso de Paula, una indígena quechuahablante de los

106 Andes peruanos, que había estudiado para ser maestra de lengua y literatura de educación secundaria y había recibido una beca para estudiar una maestría en antropología; la autora problematiza la literacidad académica, a la luz de tres aspectos: epistemología, identidad y poder. El primero se relaciona con lo que implica apropiarse de las formas más sofisticadas y elocuentes, que incluyen la polifonía y la intertextualidad como estrategias formales en la escritura académica. El segundo se refiere al costo y la incomodidad para Paula de usar este tipo de estrategias discursivas y de asumir un rol discursivo, en donde "yo" se diluye, más aún si se tiene en cuenta su origen. $Y$ en lo referido al poder se explica que efectivamente estos usos discursivos se constituyen en formas dominantes de construcción de conocimiento, siguiendo a Fairclough (1995). Se trata entonces de analizar "cómo la literacidad académica constituye un discurso construido históricamente con claros efectos ideológicos" (Zavala, 2009b, p. 351).

\section{Función epistémica de la lectura}

La función epistémica de la lectura se relaciona con el hecho de que quienes forman parte de comunidades científicas hablan y escriben textos que pueden ser comprendidos por sus miembros; además, implica que se entienda cómo se construye y comunica el conocimiento en las disciplinas. De ahí que la lectura y la escritura se constituyan en dispositivos fundamentales en el ingreso, acceso y desempeño en una 
comunidad discursiva. ${ }^{4}$ Por esto, en algunas investigaciones se aborda el papel de la lectura crítica en disciplinas específicas; en este apartado se reseñan algunas (Bazerman, 1988; Carranza, Arce, Leyva-Salas y Leyva-González, 2009; Haas, 1994; Moreno, Puerta, Cuervo y Cuéllar, 2016; Ozuru, Dempsey, McNamara, 2009; Peredo Merlo, 2016; Tapia, Ávila, Navarro y Bazerman, 2016; van Lacum, Ossevoort, Buikema y Goedhart, 2012).

Bazerman (1988) en un trabajo pionero estudió las estrategias de lectura de los científicos, para lo cual entrevistó a siete físicos y los observó mientras leían artículos. Se encontró que los físicos leen de forma selectiva, con frecuencia se saltan partes enteras del texto, todo depende de los propósitos de lectura: ya sea para ampliar conocimiento, lo cual supondría una lectura menos crítica, o ya sea para movilizar partes del artículo para su propio trabajo, lo cual supondría una lectura crítica. Según Bazerman (1988) las estrategias de lectura de los científicos dependen de lo que denomina "esquema de propósito cargado" (purpose-laden schema). Se trata de un "«mapa personal» que le proporciona un marco al lector para comprender el artículo" (p. 243). También se explica que la lectura conlleva diferentes actividades: leer fragmentos para informarse, adicionar esta información a los conocimientos previos, leer con propósitos particulares, evaluar mientras se lee, modificar la lectura dependiendo de los conocimientos previos, la experiencia y los propósitos, y la relevancia de los textos objeto de lectura para el trabajo del lector experto. Por esa misma dirección, se encuentra el estudio de Haas (1994), que asume la lectura y la escritura como actos retóricos "como el aprendizaje de los patrones de conocer y de comportarse con respecto a los textos dentro de un campo disciplinario" (p. 44). A partir del análisis de las prácticas de lectura de una estudiante llamada Eliza, que fue estudiada alrededor de cuatro años en los que estuvo en la universidad, se encontró que ella desarrolló un estilo de lectura más complejo gracias a su nivel de familiarización con el campo disciplinario.

Carranza et al. (2009) evaluaron el grado de desarrollo de lectura crítica de informes de investigación clínica en estudiantes de pregrado de octavo semestre en tres escuelas de medicina, para lo cual se aplicó un cuestionario constituido por ocho informes y 150 reactivos. Se encontró un bajo grado de desarrollo en este tipo de lectura, atribuida en especial a que predomina el consumo de información y su memorización. De otro lado, Moreno et al. (2016) analizaron una clase en la que se enseñaba a leer críticamente literatura científica. Se focalizaba la lectura como una práctica discursiva que desempeña un papel clave en el aprendizaje de textos disciplinarios, $y$, por ende, del método científico.

\footnotetext{
${ }^{4}$ Swales (1990) explica que este tipo de comunidad comparte unas prácticas y necesidades comunicativas, usan unos textos particulares y tienen unos objetivos que prevalecen en el desarrollo y mantenimiento de sus características discursivas.
} 
Ozuru et al. (2009) examinaron cómo las características del texto (cohesión) y las diferencias individuales (habilidad y conocimientos previos) contribuyen a la comprensión de textos de biología. Los estudiantes universitarios de bajos y altos niveles de conocimientos de biología leyeron dos textos de biología, uno de los cuales era alto en la cohesión y el otro bajo en la cohesión. Los dos grupos fueron similares en la habilidad de lectura. No hubo evidencia de que la lectura de textos de alta cohesión facilite más inferencias basadas en información textual que ayuden a los lectores a contestar preguntas. Se encontró que al leer ciencia, los lectores no tienen un modelo mental suficientemente desarrollado que represente las relaciones conceptuales globales entre los conceptos relevantes. Así mismo, el hecho de que el texto sea más cohesivo favorece que los lectores dependan menos de los conocimientos previos. En otro estudio, también en ciencias, Peredo Merlo (2016) presentó los resultados de una investigación sobre algunos aspectos de formación de investigadores en programas de doctorado. Se planteó que la identidad lectora se correlaciona con la identidad disciplinaria, de manera que los contextos en los cuales se desarrollan las prácticas cobran relevancia. Se concluyó que en un proceso de formación de investigadores se construye una identidad académica, lo cual supone del profesor ser un "mediador que introduce al aprendiz en un ambiente en el que se produce conocimiento, al mismo 108 tiempo que se aprende, puesto que se habla, se escribe y se lee sobre ciencia" (p. 52).

En el estudio de Tapia et al. (2016) se plantean los hitos, las causas, las actividades académicas y las disciplinas involucradas en el desarrollo de los estudios de lectura y escritura en latinoamérica. Para ello, se entrevistaron ocho académicos de diferentes países. Se concluyó que las disciplinas y las influencias son eclécticas. Los estudios en este campo incluyen diferentes tendencias desde educación, psicología educativa y lingüística. Por último, van Lacum et al. (2012) estudiaron el modo en que veinte estudiantes de ciencias biológicas analizan la estructura retórica de artículos de investigación y se compararon sus respuestas con las de dos expertos. Especialmente, en la justificación y las conclusiones, se encontró que los expertos tienen "conciencia retórica" de la estructura de los artículos. Mientras, los estudiantes no poseen este conocimiento. Igualmente, para los expertos la comprensión de la parte conceptual, la argumentación, la validez de la argumentación, el manejo de términos técnicos, la comprensión de los experimentos y la interpretación de los datos, les permiten seguir unas líneas de razonamiento sobre los artículos que leen. Otra diferencia importante tiene que ver con los propósitos de lectura, los estudiantes leen porque se les ha asignado, mientras que el experto lee con el objetivo de incorporar información a su investigación. 
Investigaciones sobre hábitos y estrategias de lectura

Entre los estudios sobre hábitos de lectura se encontraron los siguientes: Shankar, Dubey, Mishra y Upadhyay (2008); Cornejo, Roble, Barrero y Martin (2012); AguilarPérez, Cruz Covarrubias y Aguilar-Cruz (2014). En Shankar et al. (2008) se describen los hábitos de lectura de estudiantes de medicina en el Colegio de Ciencias Médicas de Manipal, durante los tres primeros semestres; así mismo, se obtiene información sobre su opinión acerca de la inclusión de las humanidades médicas en su currículo. En esa misma línea, están los estudios de Cornejo et al. (2012) y Aguilar-Pérez et al. (2014); en el primero se indaga sobre los hábitos de estudiantes de Ingeniería de la Universidad de Buenos Aires y en el segundo, sobre hábitos de estudiantes del Centro Universitario de Ciencias Económico Administrativas de la Universidad de Guadalajara. En los tres estudios se documenta qué tipo de literatura de ficción o no ficción leen los estudiantes y qué tipo de magazines, revistas y periódicos.

En cuanto a las estrategias de lectura se encontraron los estudios de Ochoa y Aragón (2005), Aragón y Caicedo (2009). Los primeros estudiaron el funcionamiento metacognitivo de estudiantes universitarios al leer artículos científicos y su nivel de comprensión. Se encontró una correlación significativa entre estos dos aspectos: a mayor nivel de funcionamiento, mayor nivel de comprensión de lectura. Igualmente, el conocimiento previo como factor importante en la comprensión también explica el hecho de que algunos estudiantes que se muestran poco regulados tienen buena comprensión, esto coincide con el estudio de Pressley y Afflerbach (1995, citados en Ochoa y Aragón, 2005) en el que se explica que los estudiantes no necesitan hacer grandes esfuerzos, porque tienen esquemas conceptuales que les permiten integrar con facilidad los conceptos de los artículos que leen, hacer inferencias y plantearse hipótesis adecuadas. De otra parte, Aragón y Caicedo (2009) presentan una revisión de estudios que contribuye desde la psicología educativa y cognitiva, analizaron específicamente reportes de investigación y ensayos teóricos publicados entre 1996 y 2008 en torno a la enseñanza de estrategias metacognitivas para el mejoramiento de la comprensión de lectura. Se plantean los aportes a nivel metodológico de las investigaciones revisadas y los objetivos que apuntan a: 1) medir la eficacia de las intervenciones, 2) enseñar habilidades para el mejoramiento de comprensión de lectura, 3) determinar la eficacia de diferentes procedimientos de instrucción, y 4) explorar la relación entre factores motivacionales y cognitivos. 


\section{DISCUSIÓN Y CONCLUSIÓN}

El análisis de los datos muestra que hay diversidad en relación con los intereses investigativos, que comprenden la lectura académica asociada a materiales, propósitos, escenarios y disciplinas. La lectura se constituye en parte fundamental de una variedad de prácticas culturales que se dan en la sociedad (Guthrie, Schafer, Wang y Afflerbach, 1995). En relación con los estados del arte que anteceden a esta investigación se encuentran coincidencias con relación a que existen diferentes perspectivas teóricas que subyacen a las prácticas -enfoque lingüístico-textual, análisis del discurso, enfoque sociocultural, enfoque semántico-comunicativo- (Uribe y Camargo, 2012), lo cual evidencia que las prácticas investigativas en torno a la lectura académica tienen de fondo marcos conceptuales diferenciados y reflejan esa diversidad en las influencias investigativas (Tapia et al. 2016). Igualmente, se destacan dos tendencias de abordaje de las prácticas de lectura, estas son: la alfabetización académica y la literacidad académica. La primera, como se dijo inicialmente, se centra en los procesos de enseñanza que se ponen en marcha para que los estudiantes accedan y se desarrollen en culturas escritas disciplinarias, mientras la segunda incluye estudios etnográficos y comprende que las 110 prácticas letradas son socioculturalmente diversas (Hernández-Zamora, 2009; Montes y López, 2017); prácticas letradas ubicadas en diferentes situaciones comunicativas y contextos sociales (el trabajo, la escuela, la familia, las comunidades, el ejercicio de la ciudadanía). En esa medida, son precisamente los elementos contextuales los que aparecen en las prácticas de lectura de modo diferenciado, dado que en cada contexto existen reglas sociales que regulan la adquisición, las funciones y el uso de la lectura (Hull, 1993).

De acuerdo con lo anterior, se hacen necesarios estudios en los cuales se analice el papel de los docentes universitarios, puesto que si bien algunos tienen la preocupación por los procesos de lectura (y escritura) de sus estudiantes, otros parten de la idea de que a través de la exposición a los textos que formulan para ser leídos, es suficiente para que el estudiante pueda apropiarse de los modos de interpretar y criticarlos dentro de su comunidad disciplinaria. Por ello, como lo plantea Carlino (2013b):

Necesitamos comprender las prácticas de enseñanza en detalle, los tipos de interacciones que ocurren, las intervenciones de los profesores que favorecen $(\mathrm{u}$ obstaculizan) que los alumnos lean y comprendan lo leído, las acciones docentes que llevan a que los estudiantes escriban para aprender, y no solo para ser evaluados en las asignaturas, las mediaciones del profesor para que sus estudiantes aprendan a escribir como se les requiere. Necesitamos describir cuáles son los tiempos que se dedican en clase a discutir colectivamente lo leído y 
lo escrito, las consignas dadas, la forma de entrelazar lectura, escritura, contenido disciplinar y comprensiones de los alumnos, cómo el docente regula las tareas en torno a lo escrito, etc. (pp. 23-24).

De ahí que la lectura en el ámbito universitario deje de ser un tema que atañe únicamente a los estudiosos del lenguaje y que reclama la participación de los docentes de las diferentes disciplinas y la reflexión sobre la articulación de las prácticas de lectura y escritura a través del currículo. Esta premisa hace evidente que los enfoques de alfabetización y literacidad académica requieren del diálogo que permita entender que cada disciplina posee unos géneros especializados, un sistema de códigos, tradiciones de comunicación, estándares de calidad, normas, dominio de habilidades discursivas y marcos epistemológicos propios.

Al leer en una disciplina, los estudiantes adquieren una práctica para abordar sus textos, esto es, "la capacidad de responder a situaciones retóricas nuevas y recurrentes en la construcción, interpretación, uso y aprovechamiento de las convenciones del género, enraizadas en culturas disciplinares específicas y en prácticas para lograr fines profesionales" (Bhatia, 2004, p. 144). Estudios como los reseñados en las categorías 3 y 5 muestran la necesidad de que sean más prolíficas las investigaciones que indagan por la función epistémica de la lectura y la escritura. Es decir, aquellas en las que se considere que la lectura y la escritura no son prácticas génericas, sino que toman forma y están determinadas por los valores y las convenciones de la disciplina (Lillis, 2001; Street, 2004). Entonces, leer y escribir suponen la formación y la integración de los estudiantes en espacios en los que los textos y las prácticas discursivas les permitan asumir su identidad profesional.

De otra parte, frente a los estudios que se han realizado en el marco de la literacidad académica, prevalecen más los que abordan las prácticas de escritura y no los de lectura. Es decir, que habría necesidad de que se hicieran investigaciones sobre cómo se configuran las prácticas de lectura en contextos disciplinarios específicos y cómo son esos modos de apropiación de la lectura por parte de los estudiantes. Investigaciones que permitan entender que el ingreso y el fortalecimiento en un campo disciplinario, en el cual la actividad de lectura se realiza guiada por unos propósitos específicos, así mismo, está ligada a los roles que ejercen docentes y estudiantes en un contexto disciplinario, en el cual son determinantes las estrategias pedagógicas y didácticas que se emplean en la enseñanza. Igualmente, se requieren estudios que vinculen las prácticas letradas al currículo, a las apuestas formativas de las instituciones y a la gestión de programas que se ocupen de la lectura y la escritura en el ámbito universitario. 


\section{REFERENCIAS}

Aguilar-Pérez, P., Cruz Covarrubias, L., y Aguilar-Cruz, P. (2014). El consumo editorial. Hábito de lectura en universitarios del Centro Universitario de Ciencias Económico Administrativas de la Universidad de Guadalajara. Contextos Educativos. Revista de Educación, (17), 109-122. doi: http://dx.doi.org/10.18172/con.2596

Aliagas, C., Castellà, J., y Cassany, D. (2009). "Aunque lea poco, yo sé que soy listo". Estudio de caso sobre un adolescente que no lee literatura. Ocnos: Revista de Estudios sobre Lectura, (5), 97-116.

Aragón, L., y Caicedo, A. (2009). La enseñanza de estrategias metacognitivas para el mejoramiento de la comprensión lectora. Estado de la cuestión. Pensamiento Psicológico, 5(12), 125-138.

Barton, D. (1994). The social impact of literacy. En L. Verhoeven (Ed.), Functional Literacy. Theoretical Issues and Educational Implications (pp. 185-197). Amsterdam: John Benjamins.

Barton, D., y Hamilton M. (1990). Researching Literacy in Industrialised Countries: Trends and Prospects. (UIE Report 2). Hamburgo: Unesco Institute for Education.

112 Barton, D., y Hamilton, M. (1998). Local Literacies. Reading and Writing in one Community. Londres, Inglaterra: Routledge.

Barton, D., y Hamilton, M. (2000). Literacy practices. En D. Barton, M. Hamilton y R. Ivanič (Eds.), Situated literacies: Reading and writing in context (pp. 7-15). Londres, Inglaterra: Routledge.

Bazerman, C. (1988). Shaping knowledge. The genre and activity of the experimental article in science. Madison: University of Wisconsin Press.

Bazerman, C., Little, J., Bethel L., Chavkin, T., Fouquette, D., y Garufis, J. (2005). Reference Guide to Writing across the Curriculum. Indiana: Parlor Press.

Berkenkotter, C., y Huckin, T. (1995). Genre Knowledge in Disciplinary Communication: Culture/Cognition/Power. Hillsdale: Lawrence Erlbaum.

Bhatia, V. K. (2004). Worlds of written discourse: A genre-based view. Londres, Inglaterra: Continuum.

Carlino, P. (2001). Hacerse cargo de la lectura y la escritura en la enseñanza universitaria de las ciencias sociales y humanas. I Jornadas sobre "La lectura y la escritura como prácticas académicas universitarias", Universidad Nacional de Luján, Luján.

Carlino, P. (2002). ¿Quién debe ocuparse de enseñar a leer y a escribir en la universidad? Tutorías, simulacros de examen y síntesis de clases en las humanidades. Lectura y Vida, 23(1), 6-14. 
Carlino, P. (2003a). Alfabetización académica: Un cambio necesario, algunas alternativas posibles. Educere, 6(20), 409-420. Recuperado de http://www.saber.ula.ve/bitstream/123456789/19736/1/ articulo7.pdf.

Carlino, P. (2003b). Leer textos científicos y académicos en la educación superior: Obstáculos y bienvenidas a una cultura nueva. Uni-Pluri/Versidad, 3(2). Recuperado de http://aprendeenlinea.udea.edu.co/revistas/index.php/unip/ article/view/12289.

Carlino, P. (2004). Escribir y leer en la universidad: responsabilidad compartida entre alumnos, docentes e instituciones. En P. Carlino (Coord.), Textos en Contexto (pp. 521). Buenos Aires, Argentina: Lectura y Vida.

Carlino, P. (2005). Escribir, leer, y aprender en la universidad. Una introducción a la alfabetización académica. Buenos Aires, Argentina: Fondo de Cultura Económica.

Carlino, P. (2007). Estudiar, escribir y aprender en universidades australianas. Textura, 6(9), 11-33. Recuperado de https://docs.google.com/viewer?a=v\&pid=sites\&srcid=ZGVmYXVsdGRvbWFpbnx qb3JuYWRhc2dpY2VvbGVtfGd4Ojc2ZGVmZDYyMzhmMzVkYzk.

Carlino, P. (2009). Reading and writing in the social sciences in Argentine universities. En C. Bazerman, R. Krut, S. Null, P. Rogers y A. Stansell (Eds.), Traditions of writing research (pp. 283-296). Oxford: Routledge/Taylor \& Francis. Recuperado de https://www.aacademica.org/paula.carlino/109.pdf.

Carlino, P. (2011). Leer y escribir en las Ciencias Sociales en universidades argentinas. Contextos de Educación, (11), 1-12.

Carlino, P. (2012). Who takes care of writing in Latin American and Spanish universities? En C. Thaiss, G. Bräuer, P. Carlino, L. Ganobcsik-Williams y A. Sinha (Eds.), Writing programs worldwide: Profiles of academic writing in many places (pp. 485-498). Recuperado de http://wac.colostate.edu/books/wpww/chapter41.pdf.

Carlino, P. (2013a). Alfabetización Académica diez años después. Revista Mexicana de Investigación Educativa, 18(57), 355-381. Recuperado de https://www.comie.org.mx/revista/v2018/rmie/index.php/nrmie/article/view/250/2 50.

Carlino, P. (2013b). Investigar las prácticas de lectura y escritura en la universidad: Necesidad de un cambio de perspectiva. En iPara qué se lee y se escribe en la universidad colombiana? Un aporte a la consolidación de la cultura académica del país (pp. 21-26). Bogotá, Colombia: Pontificia Universidad Javeriana - Colciencias.

Carranza L., S., Arce, R. M., Leyva-Salas, R., y Leyva-González, F. (2009). Lectura crítica de informes de investigación clínica en estudiantes de pregrado. Rev. Med. Inst. Mex. Seguro Soc., 47(4), 431-438.

Cartolari, M., y Carlino, P. (2011). Leer y tomar apuntes para aprender en la formación docente: un estudio exploratorio. Magis, Revista Internacional de Investigación en Educación, 4(7), 67-86. 
Cassany, D. (2006). Tras las líneas. Sobre la lectura contemporánea. Barcelona, España: Anagrama.

Cassany, D. (Comp.). (2009). Para ser letrados. Voces y miradas sobre la lectura. Barcelona, España: Paidós Educador.

Cassany, D., y Morales, O. (2009). Leer y escribir en la universidad: los géneros científicos. En D. Cassany (Comp.), Para ser letrados. Voces y miradas sobre la lectura (pp. 109-128). Barcelona, España: Paidós.

Castelló, M. (2007). El Proceso de composición de textos académicos. En M. Castelló (Coord.), Escribir y comunicarse en contextos científicos y académicos (pp. 47-82). Barcelona, España: Graó.

Castelló, M. (2009). Aprender a escribir textos académicos: ¿copistas, escribas, compiladores o escritores? En J. I. Pozo y M. P. Pérez-Echeverría (Coords.), La Psicología del aprendizaje universitario: de la adquisición de conocimientos a la formación en competencias (pp. 120-133). Madrid, España: Morata.

Chalmers, D., y Fuller, R. (1996). Teaching for learning at university. Londres, Inglaterra: Kogan Page.

Chois, P., y Santacruz, M. (2010). Prácticas de lectura y escritura académicas en el programa académico de Fonoaudiología de la Universidad del Cauca. Revista Facultad Ciencias de la Salud, 12(3), 9-15.

Cisneros, M., Olave, G., y Rojas, I. (2013). Alfabetización académica y lectura inferencial. Bogotá, Colombia: ECOE.

Cook-Gumperz, J. (Ed.). (1988). La Construcción Social de la Alfabetización. Barcelona, España: Paidós.

Cornejo, J., Roble, M. B., Barrero, C., y Martín, A. M. (2012). Hábitos de lectura en alumnos universitarios de carreras de Ciencia y Tecnología. Revista Eureka sobre Enseñanza y Divulgación de las Ciencias, 9(1), 155-163.

Di Benedetto, S., y Carlino, P. (2007). Correcciones a exámenes escritos en la universidad: cómo son y para qué sirven a los alumnos. En Memorias de las XIV Jornadas de Investigación en Psicología y Tercer encuentro de investigadores en Psicología del Mercosur (pp. 273-275). Recuperado de http://newpsi.bvspsi.org.br/mapa/Argentina/2007/tomo2.pdf.

Driver, R., Asoko, H., Leach, J., Mortimer, E., y Scott, P. (1994). Constructing Scientific Knowledge in the Classroom. Educational Researcher, 23(7), 5-12. doi: $10.2307 / 1176933$

Estienne, V., y Carlino, P. (2004). Leer en la universidad: enseñar y aprender una cultura nueva. Uni-Pluri/Versidad, 4(3), 9-17. Recuperado de http://aprendeenlinea.udea.edu.co/revistas/index.php/unip/article/viewArticle/122 10.

Fairclough, N. (1995). Critical Discourse Analysis: The Critical Study of Language. Londres, Inglaterra: Longman. 
Fang, Z. (2012). Language Correlates of Disciplinary Literacy. Topics in Language Disorders, 32(1), 19-34.

Fernández, G., y Carlino, P. (2010). ¿En qué se diferencian las prácticas de lectura y escritura de la universidad y las de la escuela secundaria? Perspectivas de alumnos y profesores en Ciencias Humanas y Veterinarias. Lectura y Vida, 31(3), 619. Recuperado de https://www.aacademica.org/paula.carlino/216.

Ford, M. J., y Forman, E. A. (2006). Redefining disciplinary learning in classroom contexts. Review of Research in Education, 30, 1-32.

García Vera, N. (2011). Cultura académica y prácticas de lectura y escritura, a propósito de la formación universitaria de docentes. Pedagogía y Saberes, (34), 117-139.

Gee, J. P. (1990) Social linguistics and literacies: Ideology in Discourses. Londres, Inglaterra: Taylor \& Francis.

Gee, J. P. (2001). Reading as situated language: A sociocognitive perspective. Journal of Adolescent \& Adult Literacy, 44(8), 714-725.

González de la Torre, Y. (2011). Configuraciones de las prácticas lectoras en contextos sociales: La lectura situada en la escuela y el trabajo. Perfiles educativos, 33(133), 3050. Recuperado de http://www.iisue.unam.mx/perfiles/articulo/2011-133configuraciones-de-las-practicas-lectoras-en-contextos-sociales-la-lectura-situadaen-la-escuela-y-el-trabajo.pdf.

González de la Torre, Y. (2013). La lectura en contextos laborales y escolares. Guadalajara, México: Universidad de Guadalajara.

Guthrie, J. T., Schafer, W., Wang, Y. Y., y Afflerbach, P. (1995). Relationships of instruction to amount of reading: An exploration of social, cognitive, and instructional connections. Reading Research Quarterly, 30(1), 8-25.

Haas, C. (1994). Learning to read biology: One student's rhetorical development in college. Written Communication, 11(1), 43-84. doi: https://doi.org/10.1177/0741088394011001004

Hernández-Zamora, G. (2009). Escritura académica y formación de maestros, ¿por qué no acaban la tesis? Tiempo de Educar, 10(19), 11-40.

Hull, G. (1993). Hearing Other Voices: A Critical Assessment of Popular Views on Literacy and Work. Harvard Educational Review, 63(1), 20-50. doi: https://doi.org/10.17763/haer.63.1.u0762m971p0645t4

Ivanic, R. (1997). Writing and Identity: The discoursal construction of identity in academic writing. Amsterdam: John Benjamins.

Ivanic, R. (1998). Writing and identity: The discoursal construction of identity in academic writing. Philadelphia, PA: John Benjamins. doi: https://doi.org/10.1075/swll.5

Jones, C., Turner, J., y Street, B. (Eds.). (1999). Students writing in the university. Cultural and epistemological issues. Amsterdam/Filadelfia: John Benjamins Publishing. doi: https://doi.org/10.1075/swll.8 
Kalman, J. (2004). Saber lo que es la letra. Una experiencia de lectoescritura con mujeres de Mixquic. México: Secretaría de Educación Pública.

Lea, M., y Street, B. (1998). Student writing in higher education: an academic literacies approach. Studies in Higher Education 23(2), 157-172. doi: https://doi.org/10.1080/03075079812331380364

Lea, M., y Street, B. (2006). The "academic literacies" model: Theory and applications. Theory into Practice, 45(4), 368-377. doi: 10.1207/s15430421tip4504_11

Lillis, T. (2001). Student Writing. Access, Regulation, Desire. Londres, Inglaterra: Routledge.

Londoño V., D., y Bermúdez, H. (2017). Niveles de literacidad en jóvenes universitarios: entrevistas cualitativas y análisis sociolingüístico. RLCSNJ, 16(1), 315-330. Recuperado de http://revistaumanizales.cinde.org.co/rlcsnj/index.php/RevistaLatinoamericana/article/view/2741.

Mateos, M., Martín, E., Pecharromán, A., Luna, M., y Cuevas, I. (2008). Estudio sobre la percepción de los estudiantes de Psicología de las tareas de lectura y escritura que realizan para aprender. Revista de Educación, 347, 255-274.

Mateos, M., y Solé, I. (2009) Synthesising information from various texts: A study of procedures and products at different educational levels. European Journal of Psychology of Education, 24(4), 435-451.

Mateos, M., Villalón, R., de Dios, M. J., y Martín, E (2007). Reading-and-writing tasks on different university degree courses: What do the students say they do? Studies in Higher Education, 32(4), 489-510. doi: https://doi.org/10.1080/03075070701476183

Moje, E. (2007). Developing Socially Just Subject-Matter Instruction: A Review of the Literature on Disciplinary Literacy Teaching. Review of Research in Education, 31(1), $1-44$.

Moje, E. (2008). Foregrounding the disciplines in secondary literacy teaching and learning: A call for change. Journal of Adolescent \& Adult Literacy, 52(2), 96-107.

Montes S., M., y López-Bonilla, G. (2017). Literacidad y alfabetización disciplinar: enfoques teóricos y propuestas pedagógicas. Perfiles educativos, 39(155), 162-178. Recuperado de http://www.iisue.unam.mx/perfiles/articulo/2017-155-literacidady-alfabetizacion-disciplinar-enfoques-teoricos-y-propuestas-pedagogicas.pdf.

Moreno, E., Puerta, C., Cuervo, C., y Cuéllar, A. (2016). Análisis crítico de literatura científica. Una experiencia de la Facultad de Ciencias de la Pontificia Universidad Javeriana. Voces y silencios. Revista Latinoamericana de Educación, 7(2), 74-97.

Narváez Cardona, E. (2012) Training experiences in reading and writing in a Colombian university: the perspective of a professor. En C. Thaiss, G. Bräuer, P. Carlino, L. Ganobcsik-Williams y A. Sinha (Eds.), Writing Programs Worldwide: Profiles of Academic Writing in Many Places (pp. 147-156). California: The WAC Clearinghouse. 
Narváez, E., y Cadena, S. (2009). La enseñanza de la lectura académica: un objeto de formación docente. Lectura y Vida, 30(1), 56-67.

Narváez, E., y Chois, M. (2012). La lectura y la escritura académica en la universidad colombiana: desafíos para la orientación de políticas educativas en el marco de una investigación interuniversitaria. Lenguaje, 40(2), 285-313.

Narvaja de Arnoux, E., Di Stefano, M., y Pereira, C. (2002). La lectura y la escritura en la Universidad. Buenos Aires, Argentina: Eudeba.

Ochoa, S., y Aragón, L. (2005). Comprensión lectora y funcionamiento metacognitivo en estudiantes universitarios. Universitas Psychologica, 4(2), 179-196.

Ozuru, Y., Dempsey, K., y McNamara, D. S. (2009). Prior knowledge, reading skill, and text cohesion in the comprehension of science texts. Learning and Instruction, 19(3), 228-242. doi: 10.1016/j.learninstruc.2008.04.003

Parodi, G. (Ed.). (2010). Alfabetización académica y profesional en el siglo XXI: leer y escribir desde las disciplinas. Chile: Ariel.

Peredo Merlo, A. (2002). De lectura, lectores y contextos. Una aproximación sociocognoscitiva. Guadalajara, México: Universidad de Guadalajara.

Peredo Merlo, A. (2016). Lectura y ciencia en diversos posgrados y disciplinas. Revista de la Educación Superior, 45(180), 41-54. doi: https://doi.org/10.1016/j.resu.2016.07.001

Pérez, M., y Rincón, G. (Coords.) (2013). Para qué se lee y se escribe en la universidad colombiana?: un aporte a la consolidación de la cultura académica del país. Bogotá, Colombia: Colciencias - Pontificia Universidad Javeriana.

Pérez, M., y Rodríguez, A. (2013). ¿Para qué se lee y se escribe en la universidad colombiana? caracterización de prácticas de lectura y escritura en 17 universidades. REDU, Revista de Docencia Universitaria, 11(1), 137-160. doi: https://doi.org/10.4995/redu.2013.5595

Quintana, H., García-Arroyo, M., Arribas, M., y Hernández, C. (2010). La alfabetización académica en las instituciones de educación superior en Puerto Rico en el siglo XXI. En G. Parodi (Ed.), Alfabetización Académica y profesional en el siglo XXI: Leer y escribir desde las disciplinas (pp. 21-47). Chile: Ariel.

Quintero, G., y Duque, A. (2010). La lectura y la formación profesional. Revista Colombia Hechos y Proyecciones del Lenguaje, (19), 176-198.

Rincón, G., y Gil, J. (2010). Las prácticas de lectura y de escritura académicas en la Universidad del Valle: tendencias. Lenguaje, 38(2), 387-419.

Rockwell, E., y Mercado, R. (1988). La práctica docente y la formación de maestros. Investigación en la Escuela, (4), 65-78.

Russell, D. (2002). Writing in the academic disciplines. A curricular history. Carbondale y Edwardsville: Southern Illinois University Press.

Salazar, A., Sevilla, O., González, B., Mendoza, C., Echeverri, A., Quecán, D., ... Lozano, M. (2015). Lectura y escritura en la universidad: contribución para reconstruir una 
historia. Magis, Revista Internacional de Investigación en Educación, 8(16), 51-70. doi: http://dx.doi.org/10.11144/Javeriana.m8-16.leuc

Shankar, R., Dubey, A., Mishra, P., y Upadhyay, D. (2008). Reading Habits and Attitude toward Medical Humanities of Basic Science Students in a Medical College in Western Nepal. Teaching and Learning in Medicine, 20(4), 308-313. doi: 10.1080/10401330802384599

Solé, I., Mateos, M., Miras, M., Martín, E., Castells, N., Cuevas, I., y Gràcia, M. (2005). Lectura, escritura y adquisición de conocimientos en Educación Secundaria y Educación Universitaria. Infancia y Aprendizaje, 28(3), 329-347. doi: https://doi.org/10.1174/0210370054740241

Stockdill, D., Learned, J., y Moje, E. B. (2011). Integrating reading strategies and knowledge building in adolescent literacy instruction. En A. E. Farstrup y J. Samuels (Eds.), What Reading Research Has to Say to Reading Instruction (pp. 159185). Newark: International Reading Association.

Street, B. V. (1984). Literacy in theory and practice. Cambridge: Cambridge University Press.

Street, B. V. (1993). Introduction: The New Literacy Studies. En B. V. Street (Ed.), Crosscultural approaches to literacy (pp. 1-21). New York: Cambridge University Press.

Street, B. V. (2004). Los nuevos estudios de literacidad. En V. Zavala, M. Niño-Murcia y P. Ames (Eds.), Escritura y sociedad: nuevas perspectivas teóricas y etnográficas (pp.81107). Lima, Perú: Red para el Desarrollo de las Ciencias Sociales en el Perú.

Swales, J. M. (1990). Genre analysis: English in academic and research settings. Cambridge: Cambridge University Press.

Tapia L. M., Ávila N., Navarro, F., y Bazerman, C. (2016). Milestones, Disciplines and the Future of Initiatives of Reading and Writing in Higher Education: An Analysis from Key Scholars in the Field in Latin America. Ilha do Desterro, 69(3), 189-208. doi: https://dx.doi.org/10.5007/2175-8026.2016v69n3p189

Tejada, H., y Vargas F., A. (2011). Hacia una integración de la literacidad crítica, la literacidad funcional y la literacidad cultural. Lenguaje, 35(2), 197-219. Recuperado de http://revistaingenieria.univalle.edu.co/index.php/lenguaje/article/view/4857.

Uribe, G., y Camargo, Z. (2012). Prácticas de lectura y escritura académicas en la universidad colombiana. Magis. Revista Internacional de Investigación en Educación, 3(6), 317-341. Recuperado de http://revistas.javeriana.edu.co/index.php/MAGIS/article/view/3543.

van Lacum, E., Ossevoort, M., Buikema H., y Goedhart M. (2012). First Experiences with Reading Primary Literature by Undergraduate Life Science Students. International Journal of Science Education, 34(12), 1795-1821. doi: https://doi.org/10.1080/09500693.2011.582654 
Verhoeven, L. (1994). Modeling and promoting functional literacy. En L. Verhoeven (Ed.), Functional Literacy: Theoretical issues and educational implications (pp. 3-33). Amsterdam: John Benjamins Publishing Company.

Wineburg, S. S. (1991). Historical problem solving: A study of the cognitive process used in the evaluation of documentary and pictorial evidence. Journal of Educational Psychology, 83(1), 73-87.

Wineburg, S. S. (1998). Reading Abraham Lincoln: An expert/expert study in the interpretation of historical texts. Cognitive Science, 22(3), 319-346. doi: https://doi.org/10.1207/s15516709cog2203_3

Wineburg, S. S. (2001). Historical Thinking and Other Unnatural Acts: Charting the Future of Teaching the Past. Filadelfia: Temple University Press.

Zavala, V. (2002) Desencuentros con la escritura. Escuela y comunidad en los andes peruanos. Lima, Perú: Red para el desarrollo de las ciencias sociales en el Perú.

Zavala, V. (2009a). La literacidad o lo que la gente hace con la lectura y la escritura. En D. Cassany (Ed.), Para ser letrados. Voces y miradas sobre la lectura (pp. 23-35). Barcelona, España: Paidós.

Zavala, V. (2009b). “QQuién está diciendo eso?” Literacidad académica, identidad y poder en la educación superior. En J. Kalman y B. Street (Coords.), Lectura, escritura y matemáticas como prácticas sociales: diálogos con América latina (pp.348363). México: Siglo XXI-CREFAL.

Zavala, V., Niño-Murcia M., y Ames, P. (Eds.). (2004). Escritura y sociedad. Nuevas perspectivas teóricas y etnográficas. Lima, Perú: Red para el desarrollo de las ciencias sociales en el Perú.

\section{SOBRE LA AUTORA}

\section{Emilce Moreno Mosquera}

Licenciada en Español y Filología Clásica, y Magíster en Lingüística de la Universidad Nacional de Colombia. Doctora en Educación de la Universidad Pedagógica Nacional. Actualmente se desempeña como profesora en la Facultad de Educación de la Pontificia Universidad Javeriana. Es miembro de los grupos de investigación "Pedagogías de la Lectura y la Escritura" de esta última universidad y del grupo "Lingüística y Educación" de la Universidad Nacional. Sus áreas de interés académico son: Enseñanza y aprendizaje de la lectura y la escritura académica, a través de las disciplinas y como prácticas situadas, desde el enfoque de literacidad académica y disciplinaria; Escritura académica en posgrados; Didáctica aplicada a la enseñanza de la lengua materna, y Enseñanza de la lectura y la escritura en los diferentes niveles de educación. Correo electrónico: moreno-e@javeriana.edu.co 